\title{
Un Cas de Lupus Erythemateux Dissemine (LED) Revele par une Anemie Chronique au Service d'hematologie Clinique du CHU de Yopougon
}

\author{
Diakite Mamady, \\ Service d'Hématologie clinique du CHU de Yopougon \\ Service d'Hématologie, Hôpital National Donka, CHU de Conakry \\ Kouakou Boidy, \\ Service d'Hématologie clinique du CHU de Yopougon \\ Kante Ansoumane Sayon, \\ Service d'Hématologie, Hôpital National Donka, CHU de Conakry \\ Kamara Ismael, \\ Silue Dohomas Alexis, \\ Service d'Hématologie clinique du CHU de Yopougon \\ Keita Fatimata, \\ Service de Dermatologie-MST de l'Hôpital national Donka \\ CHU de Conakry \\ Danho Nanho Clotaire, \\ Tolo Aissata, \\ Sanogo Ibrahima, \\ Service d'Hématologie clinique du CHU de Yopougon \\ URL:http://dx.doi.org/10.19044/esj.2019.v15n12p96 \\ Les auteurs rapportent un cas de lupus érythémateux disséminé révélé \\ par une anémie chronique. IL s'agissait d'une patiente de 29 ans présentant \\ une fièvre au long cours, des lésions cutanées et articulaires d'évolution \\ chronique avec à l'hémogramme une anémie hémolytique isolée hypochrome \\ microcytaire. Le diagnostic de LED a été retenu trois années après le début \\ de la symptomatologie devant sept critères sur 11 de L'American \\ Rheumatology Association (ARA) dont le bilan immunologique positif \\ (anticorps antinucléaires et DNA natif). Cette observation montre l'intérêt \\ d'évoquer le LED, tout en recherchant les signes chez une femme jeune \\ présentant une symptomatologie multiple et variée avec les signes d'atteintes \\ cutanée, rénale, ostéo-articulaire et hématologique.
}

\section{Résumé}


Mots-clés : Anémie Hémolytique, Lupus Erythémateux Disséminé, Afrique subsaharienne

\title{
A Case of Lupus Erythematosus Dissemina (LED) Revealed by a Chronic Anemia in the Clinical Hematology Department of Yopougon CHU/Abidjan
}

\author{
Diakite Mamady, \\ Service d'Hématologie clinique du CHU de Yopougon \\ Service d'Hématologie, Hôpital National Donka, CHU de Conakry \\ Kouakou Boidy, \\ Service d'Hématologie clinique du CHU de Yopougon \\ Kante Ansoumane Sayon, \\ Service d'Hématologie, Hôpital National Donka, CHU de Conakry \\ Kamara Ismael, \\ Silue Dohomas Alexis, \\ Service d'Hématologie clinique du CHU de Yopougon \\ Keita Fatimata, \\ Service de Dermatologie-MST de l'Hôpital national Donka \\ CHU de Conakry \\ Danho Nanho Clotaire, \\ Tolo Aissata, \\ Sanogo Ibrahima, \\ Service d'Hématologie clinique du CHU de Yopougon
}

\begin{abstract}
The authors report one case of systemic lupus erythematosus revealed by chronic anemia. This was a 29 -year-old patient with long-term fever, chronic skin and joint lesions with isolated hypochrome microcytic haemolytic anemia on the hemogram. The diagnosis of SLE was made three years after the onset of symptomatology based on seven of the American Rheumatology Association's (ARA) criteria out of 11, including positive immunological status (antinuclear antibodies and native DNA). This observation shows the interest of evoking SLE, while looking for signs in a
\end{abstract}


young woman with multiple and varied symptoms with signs of skin, kidney, osteoarticular and hematological disorders.

Keywords: Haemolytic Anemia, Systemic Lupus Erythematosus, Subsaharan Africa

\section{Introduction}

Archétype de la maladie auto-immune non spécifique d'organe, le lupus érythémateux systémique (LES) est un syndrome caractérisé cliniquement par l'association de manifestations protéiformes et biologiquement par la présence presque constante d'anticorps dirigés contre divers constituants du noyau (anticorps anti nucléaires). (James, 2014).

Ces atteintes organiques sont multiples et évoluent par poussées successives entrecoupées de période de rémission. Les manifestations cliniques initiales sont polymorphes et parfois trompeuses.

Les critères diagnostiques de la maladie lupique actuellement retenus ont été établis et corrigés en 1982, puis remis à jour en 1997 par l'American Rheumatology Association (ARA) (James, 2014 ; Hochberg, 2015). La maladie touche les femmes jeunes sept à neuf fois sur dix. La prévalence du LES est de 15 à 20 cas pour 100000 habitants (James, 2014 ; Hochberg, 2015). Les atteintes cutanées, articulaires et hématologiques sont les plus fréquentes et quasi constantes. Les atteintes cutanées sont variées allant des atteintes spécifiques lupiques et non spécifiques, l'atteinte hématologique comporte la survenue de cytopénies auto-immunes (James, 2014).

Le polymorphisme clinique révélateur peut être trompeur. Ce qui a suscité l'intérêt de rapporter un cas de lupus révélé dans la suite de l'exploration d'une anémie hémolytique chronique chez une femme de 29 ans.

\section{Observation}

Une patiente de 29 ans, ménagère, sans antécédents particuliers, nous a été référée d'un hopital de province pour la prise en charge d'une anémie chronique. Elle aurait été transfusée plusieurs fois sans succès.

Dans les antécédents familiaux, nous avons notés une notion d'anémie sévère à répétition chez la mère non explorée.

L'anamnèse retrouve un début qui remonterait à environ trois (03) ans marqué par la survenue d'une asthénie physique d'intensité progressive, des douleurs articulaires, des palpitations, de fièvre et d'œdèmes des membres inférieurs évoluant dans un contexte d'altération de l'état général, sans notion de troubles digestifs ni de signes pulmonaires.

L'épisode actuel remonterait à deux (02) semaines par l'exacerbation des mêmes symptômes ayant nécessité une nouvelle consultation dans un 
hôpital général. L'hémogramme réalisé a objectivé une anémie isolée hypochrome microcytaire avec un taux d'hémoglobine de 3,6g/l. Elle a été transfusée avec $820 \mathrm{ml}$ de concentré érythrocytaire dont le contrôle a trouvé un taux d'hémoglobine de $3,8 \mathrm{~g} / \mathrm{l}$. elle nous a donc été adressée pour une meilleure prise en charge.

L'examen clinique à l'entrée a noté une patiente subfébrile avec une température de $38,7^{\circ \mathrm{C}}$ et un performans status à 2 , associé à des œdèmes aux membres inferieurs bilatéraux, symétriques, gardant le godet, une pâleur cutanéo-muqueuse et une hyperpigmentation surmontée de micro papules en aile de papillon circonscrivant le nez. Nous avons objectivé une voussure sus ombilicale indolore expansive et impulsive à l'effort de toux. L'abdomen volumineux avec un signe de flot positif. L'auscultation pulmonaire retrouvait une diminution du murmure vésiculaire à la base du poumon droit et l'auscultation cardiaque notait une tachycardie à 126 battements/ min.

L'hémogramme a montré une anémie isolée hypochrome microcytaire avec un taux d'hémoglobine de $3,1 \mathrm{~g} / \mathrm{dl}$. La VS à la $1^{\text {ère }}$ heure était accélérée à $40 \mathrm{~mm}$. Le Métabolisme du fer était normal. Le bilan biochimique multiparamétrique retrouvait une créatinine, un taux de lactate déshydrogénase ( $\mathrm{LDH}$ ) et une $\mathrm{C}$ réactive protein (CRP) augmentées respectivement à $26 \mathrm{mg} / \mathrm{l}$ (DFG à $36,04 \mathrm{ml} / \mathrm{min}$ ) $520 \mathrm{UI}$ et $48 \mathrm{mg} / \mathrm{l}$. Le bilan hépatique était normal de même que l'ionogramme sanguin et l'urée. Le Bilan d'hémostase était normal. La goutte épaisse et la recherche de bacille de Koch étaient négatives ainsi que la sérologie rétrovirale. Aucun germe n'a été retrouvé sur les prélèvements d'urine et d'hémoculture. Le test de Coombs direct, était positif. Les anticorps anti nucléaires positifs et des autos anticorps anti DNA natifs étaient positifs avec respectivement 1280UI et 30UI.

D'autres anticorps comme : les antis Sm, anti SSA, SSB et RNP qui se rencontrent dans d'autres connectivites n'ont pu être réalisés chez notre patiente.

La radiographie pulmonaire de face montrait une pleurésie droite de faible abondance; l'échographie cardiaque, une péricardite minime et l'échographie abdomino-pelvienne, une ascite de moyenne abondance associée à une souffrance rénale stade II et une hernie de la ligne blanche en sus ombilicale.

Au terme de ce bilan clinique et para clinique, le diagnostic de lupus érythémateux disséminé a été retenu devant $7 / 11$ critères de l'American Rheumatology Association qui sont: l'érythème malaire, l'arthrite, la Polysérite, la protéinurie des $24 \mathrm{H}$ supérieure $0,5 \mathrm{~g} / \mathrm{l}$, la présence d'une anémie hémolytique, la positivité des anticorps anti nucléaires et anti DNA natif. 
Il est associé à une atteinte rénale (devant l'insuffisance rénale modérée et les signes de souffrance rénale retrouvée à l'échographie) et une hernie de la ligne blanche.

La prise en charge thérapeutique a consisté à un support transfusionnel fait de trois poches de $350 \mathrm{cc}$ de concentrés érythrocytaires phénotypés, une corticothérapie à dose dégressive en raison de $1,5 \mathrm{mg} / \mathrm{kg} / \mathrm{jour}$ avec les mesures d'accompagnement (déparasitage, supplémentation en calcium et potassium, apport d'antiacide, un régime hyposodé et hypoglucidique, surveillance du poids, de la tension artérielle, de la glycémie et des autres paramètres vitaux).

Elle a bénéficié d'une prise en charge multidisciplinaire regroupant les hématologues, les néphrologues, les chirurgiens digestifs et les rhumatologues.

L'évolution a été marquée par une normalisation de l'hémogramme et du bilan rénal après 2 mois de traitement, une régression importante des signes cutanés avec résorption des œdèmes.

\section{Discussion}

Le lupus érythémateux disséminé est une pathologie chronique qui se présente avec une symptomatologie polymorphe avec atteintes multivariées dont entre autres, cutanée, rénale et hématologique. (James, 2014). L'âge de survenue chez notre patiente et le sexe concordent avec les données de la littérature qui stipulent qui que c'est une pathologie fréquente chez le sexe féminin et la tranche d'âge de survenue la plus fréquente chez le sujet adulte qui est de 20 à 40 ans. (Bader Menuier et coll, 2003 ; Fleur et coll, 2013).

L'érythème malaire en aile de papillon circonscrivant le nez retrouvé chez la patiente et épargnant le sillon naso labial est également identifié dans les critères de l'American Rheumatology Association. (HAS, 2010, Guidelines SLE, 1999 ; Orphanet, 2008).

La pathologie est caractérisée par un processus inflammatoire chronique. La vitesse de sédimentation est constamment élevée au cours des poussées par contre, la protéine $\mathrm{C}$ réactive est pas ou peu augmentée $(<50$ $\mathrm{mg} / \mathrm{l})$ sauf en cas d'atteinte des séreuses ou d'infection, où l'élévation peut être importante. (Kelly et coll, 2002 ; Mallesson et coll 1997).

Le bilan inflammatoire de notre patiente retrouve un syndrome inflammatoire avec un taux de CRP et VS respectivement de $48 \mathrm{mg} / \mathrm{l}$ et $40 \mathrm{~mm}$ à la $1^{\text {ère }}$ heure concordait avec ces données.

Concernant l'hémogramme, il objective une anémie isolée hypochrome microcytaire avec un taux d'hémoglobine de 3,1g/dl. Il s'agit d'une anémie hémolytique auto-immune qui a été la circonstance de découverte du LED (Scofiel et coll, 2003).

Les maladies auto-immunes non spécifiques d'organe dont le lupus érythémateux disséminé et syndrome de Gougérot Sjogrën, s'accompagnent 
fréquemment d'une cytopénie. Celle-ci peut être la conséquence d'action directe des anticorps spécifiques d'autos antigènes cibles exprimés par les cellules hématopoïétiques ou leurs précurseurs. Il est donc habituel de rechercher ces maladies auto-immunes non spécifiques d'organes devant toutes manifestations d'auto-immunité touchant une lignée hématopoḯtique. La fréquence avec laquelle ces manifestations sont retrouvées au cours du LED reste encore inexpliquée. Le rôle de facteurs génétiques dans la survenue des cytopénies auto-immunes est suggéré par les résultats d'une analyse de liaison par tour de génome. La stratification phénotypique notamment réalisée sur la présence d'une thrombopénie ou d'une anémie hémolytique autoimmunes, montre l'existence d'une liaison significative de ces manifestations avec deux locus localisés respectivement en $11 \mathrm{p} 14$ et en 11p13(Lee et coll, 2004 ; Daniel, 2011). Les gènes associés à ces manifestations hématologiques ne sont pas encore identifiés.

Des anticorps anti nucléaires étaient positifs supérieurs à 1280UI alors que des autos anticorps anti DNA natif revenait positif supérieur à 30. Les facteurs antinucléaires sont positifs chez 94 à $100 \%$ des patients porteurs de LED mais ont une mauvaise spécificité et sont retrouvés chez $5 \%$ de la population générale; la spécificité de ce test augmente avec l'augmentation des taux de $35 \%$ pour un taux faible de $1 / 20^{\circ}$ à plus de $95 \%$ pour des taux $\geq$ $1 / 1280^{\circ}$ respectivement.

Les anticorps anti $\mathrm{Sm}$, peu sensibles mais très spécifiques du LED et les anticorps anti SSA, SSB et RNP qui se rencontrent dans d'autres connectivites. Les anticorps anti Sm se rencontrent dans 11 à 45\% des LED pédiatriques, les anticorps anti SSA dans 14 à 40\%. (Daniel, 2011). Les anticorps anti RNP dans 11 à $47 \%$ des cas. Une diminution des fractions C3 ou du C4 du complément est présente chez 65 à $91 \%$ des enfants. L'association d'une hypocomplementémie et d'un titre élevé d'anticorps anti DNA natifs a une valeur prédictive positive de $100 \%$ pour le diagnostic de LED. (Daniel, 2011 ; Raphaelle, 2014). Ces données sont rapportées dans la littérature mais n'ont pas pu être recherchées chez notre patiente.

L'atteinte rénale est la plus importante dans les séries africaines. (Ka MM et coll, 1998). Elle est reconnue par la plupart des auteurs comme étant la plus grande pourvoyeuse de mortalité au cours du lupus. Cependant dans certaines séries occidentales (Kelly et coll, 2002;Raphaelle, 2014), la mortalité par atteinte rénale diminue, alors que celle par atteinte cardiovasculaire gagne du terrain ; ceci est dû aux possibilités d'hémodialyse et de transplantations pour les malades présentant une insuffisance rénale chronique.

\section{Conclusion}

Le LED demeure une maladie multi systémique potentiellement sévère dont la manifestation clinique est polymorphe. Il doit être évoqué chez toutes 
femmes adultes jeunes présentant des signes cutanés, articulaires avec des perturbations hématologiques et rénales.

Conflit d'intérêt: nous ne signalons aucun.

Sincères remerciements: à tous ceux qui ont participés à la prise en charge de la patiente d'une manière ou d'une autre avec une mention particulière à tout le personnel du service d'hématologie clinique du CHU de Yopougon à Abidjan.

\section{References:}

1. Bader-Menuier B, Armengaud JB, Cohat P. Presenting manifestations of systemic lupus erythematosus (SLE) in 136 childrens. Clinic Exp Rheum 2003; 21: 551.

2. Sailler L. Le lupus érythémateux disséminé, un bon pronostic si vigilance. Le quotidien du médecin. 2017;22 :1-2.

3. Fleur BB, Dudler J. Lupus érythémateux disséminé, quelle prise en charge en 2013 ? Forum Med Suisse $2013 ; 13$ :841 - 5.

4. Guidelines for referral and management of systemic lupus erythematosus in adults. Arthritis and Rheumatism. 1999 ; 42 : 178596.

5. Haute Autorité de santé (HAS). lupus érythémateux disséminé ; protocole national de diagnostic et de soins ; France ; 2017 ;21 :1-87.

6. Hochberg MC. Updating the Americain College of rheumatology revised criteria for the classification of systemic lupus erythematosus. Arthritis Rheum; 1998; 41(4):751.

7. James K, Labojka D. Nouveaux éclairages sur le LED (Lupus Erythémateux Disséminé). Newsletter SCOR Global Life. $2014 ; 1: 1961-7062$

8. Kelly JA, Thompson K, Kilpatrick J, Lam T, Nath SK, Gray-McGuire $\mathrm{C}$ et al. evidence for a succeptibility gene (SLEH1) on chromosome 11q14 for systemic lupus erythematosus families with hemolytic anemia. Proc Natl Acad Sci UA 2002 ; 99 : 11766-71

9. Amoura Z. Le lupus érythémateux disséminé. Encyclo orphanet Grand pub ; $2008 ; 82: 1-14$

10. Lee NJ, Rigby RJ, Gill H, Boye JJ, Fossati-Jmak L, Morley BJ, Vyse TJ. Multiple loci are linked with ant-red blood cell antibody production in NZB mice ; comparison with other phenotypes implies complex modes of action. Clin ExpI mmunol 2004 ; 138 : 39-46

11. Malleson PN, Sailer M, Mackinnon MJ.usefulness of antinuclear antibody testing to screen for rheumatic deseases; Arch Dis Child $1997 ; 77: 299-304$. 
12. Munoz LE, Gaipl US, Franz S, Sheriff A, Voll RE, Kalden JR et al. A disease of clearance deficiency. Rheumatology (oxford); 2005; 44:1101- 7 .

13. Raphaelle S. évaluation thérapeutique des lupus érythémateux disséminé. Clin Exp Immunol ; $2014 ; 21: 209$ - 221.

14. Ka MM, Diallo S, Kane A, Wade B, Mbengue M, Diouf B. Lupus Erythémateux Systémique au Sénégal. Med d'Afr Noire; $1998 ; 45(1): 41-5$

15. Scofiel RH, Bruner GR, Kelly JA, Kilpatrick J, Bacino D, Nath SK, et al. Thrombocytopenia identifies a severe familial phenotype implies of systemic lupus erythematosus and reveals genetic linkages at 1q22 and 11q23. Blood $2003 ; 101: 992-7$ 